\title{
Excited-state quantum phase transitions and periodic dynamics
}

\author{
G. Engelhardt.* V. M. Bastidas, W. Kopylov, and T. Brandes \\ Institut für Theoretische Physik, Technische Universität Berlin, Hardenbergstr. 36, 10623 Berlin, Germany
}

\begin{abstract}
We investigate signatures of the excited-state quantum phase transition in the periodic dynamics of the Lipkin-Meshkov-Glick model and the Tavis-Cummings model. In the thermodynamic limit, expectation values of observables in eigenstates of the system can be calculated using classical trajectories. Motivated by this, we suggest a method based on the time evolution of the finite-size system, to find singularities in observables, which arise due to the excited-state quantum phase transition.

PACS numbers: 67.85.-d, 05.30.-d, 21.60.Ev, 03.65.Sq
\end{abstract}

\section{INTRODUCTION}

Besides the well-known quantum bifurcation in the ground state, known from the Dicke model 1 and the bosonic Josephson junction [2, quantum criticality also takes place in excited states [3. A key feature of the so-called excited-state quantum phase transitions (ESQPTs), is a level clustering at critical energies, which results in a logarithmic singularity in the density of states (DOS) 3 5. This singularity is induced by a saddle point of the semiclassical energy surface [3]. Accordingly, in the thermodynamic limit the eigenstates of the quantum system also experience symmetry breaking, in the sense of being degenerate or not, depending on their energy [6].

As a consequence of the strong relation to the classical dynamics, the underlying classical dynamics drives the ESQPT, which can be considered to be a quantum manifestation of a separatrix [7.

Among the quantum signatures of the separatrix 2, 8[19], intriguing relations to spin squeezing and entanglement 20 22] have been explored. Previous works study a plethora of dynamical consequences of ESQPTs, i.e., their influence on quantum quenches [23], the relation to chaos 24], and decoherence of a qubit coupled to a system possessing an ESQPT [25]. In the context of driven quantum systems, singular behavior resembling ESQPTs appears in the quasienergy spectrum of the kicked top [26].

Experimental signatures of ESQPTs have been found in molecular systems [27, in the diverging period of a spinor Bose-Einstein condensate 28, and in microwave billiards 29]. In pioneering experiments, Oberthaler and collaborators realized the anisotropic Lipkin-MeshkovGlick (LMG) model by coupling the internal degrees of freedom of a ${ }^{87} \mathrm{Rb}$ Bose-Einstein condensate 30 32. Additionally, the striking experimental observation of Dicke superradiance in Bose gases loaded in a cavity [33, 34, opens the possibility to explore the physics of excited states, due to the high degree of control of the system. Recently, there has been a renewed interest in the experimental investigation of the Tavis-Cummings model,

*Electronic address: georg@itp.tu-berlin.de which is realized using cavity-assisted Raman transitions 35.

Motivated by these experimental highlights, in this paper we explore the signatures of the ESQPT that appear in the periodic dynamics of the LMG model and the Tavis-Cummings (TC) model. In doing so, we use the fact that expectation values in eigenstates can be calculated by a semiclassical temporal average [4, 36. In contrast to the LMG model, the classical dynamics of the TC model do not occur on a two-dimensional manifold. Therefore, it is interesting to investigate if our approach is also applicable for models with regular dynamics and a high-dimensional phase space such as the TC model.

The rest of the paper is organized as follows. In Sec. II] we introduce the models that will be discussed along the paper, their corresponding semiclassical limits and the method to calculate time-averaged expectation values. In Sec. III] we discuss our results for the LMG and TC models. In particular, we perform numerical calculations for a finite-size system to compare with the semiclassical results. Finally, in Sec. IV we discuss the application of our method to the LMG model for realistic experimental parameters. The conclusions in Sec. IV] are followed by the Appendix, where we discuss in detail the semiclassical calculations for the Tavis-Cummings model.

\section{MODELS AND METHODS}

\section{A. Hamiltonians}

The LMG model describes a set of $N$ two-level systems in a transverse field in the $z$-direction which have anisotropic interactions $[37+39]$. The Hamiltonian reads

$$
H_{\mathrm{LMG}}=-h J_{z}-\frac{1}{N}\left(\gamma_{x} J_{x}^{2}+\gamma_{y} J_{y}^{2}\right),
$$

where $J_{\alpha}=\frac{1}{2} \sum_{i}^{N} \sigma_{i}^{\alpha}$ and $\sigma_{i}^{\alpha}$, with $\alpha \in\{x, y, z\}$ denote the Pauli matrices. The parameters $\gamma_{x}$ and $\gamma_{y}$ describe the interaction strength in the $x$ and $y$ directions, respectively.

To get a well-defined thermodynamic limit, we restrict the spin Hilbert space to the subspace with maximal total angular momentum $j=N / 2$ as in Refs. [1, 4, [5]. This 
allows to uniquely describe the atomic Hilbert space by using Dicke states $|j, m\rangle$, which are the eigenstates of $J_{z}$ [0].

In the experimental realization of the LMG model in Ref. 30, 31] by coupling the internal degrees of freedom of a Bose-Einstein condensate, one is naturally restricted to $j=N / 2$ in the case of a system consisting of $N$ indistinguishable bosonic particles 32. The underlying reason is that the spatial wave functions of all bosons are identical due to condensation. This implies that the internal degrees of freedom have to be symmetric under permutation. As the Dicke states with $j=N / 2$ satisfy this condition 40, these are the physically relevant states.

The TC Hamiltonian describes an ensemble of twolevel systems with level splitting $\omega_{0}$, which are collectively coupled with strength $\lambda$ to a cavity field of frequency $\omega$

$$
H_{\mathrm{TC}}=\omega \hat{a}^{\dagger} \hat{a}+\omega_{0} J_{z}+\frac{\lambda}{\sqrt{N}}\left(\hat{a} J_{+}+\hat{a}^{\dagger} J_{-}\right),
$$

where $\hat{a}$ and $\hat{a}^{\dagger}$ are bosonic operators and $J_{ \pm}=J_{x} \pm$ $i J_{y}$ [1, 5, 41,43].

The bosonic mode is described in terms of Fock states $|n\rangle$. The TC Hamiltonian commutes with the operator $\hat{\mathcal{M}}=\hat{a}^{\dagger} \hat{a}+J_{z}$. This allows to restrict the basis of the Hilbert space $\{|n\rangle \otimes|j, m\rangle\}$ to the symmetry-adapted basis $\{|M-m\rangle \otimes|j, m\rangle\}$, where $M=n+m$ is the eigenvalue of $\hat{\mathcal{M}}[42]$.

\section{B. The semiclassical energy landscapes}

To obtain the semiclassical energy landscape $E_{\mathrm{LMG}}$ of the LMG model, we scale the Hamiltonian Eq. (1) with $j$ and consider the thermodynamic limit $j \gg 1$. In this limit, quantum fluctuations are negligible and we can define classical variables $j_{\alpha}=J_{\alpha} / j$ with Poisson bracket $\left\{j_{x}, j_{y}\right\}=j_{z}$ as in Ref. [44. By using these classical variables we define the energy landscape

$$
E_{\mathrm{LMG}}(\mathbf{j})=-h j_{z}-\frac{1}{2}\left(\gamma_{x} j_{x}^{2}+\gamma_{y} j_{y}^{2}\right),
$$

where $\mathbf{j}=\left(j_{x}, j_{y}, j_{z}\right)$. In this paper we consider the parametrization $\mathbf{j}=(\sin \theta \cos \phi, \sin \theta \sin \phi, \cos \theta)$ of the Bloch sphere in terms of the local coordinates $(\theta, \phi)$.

In the insets of Fig. 1, we depict the level sets of the classical energy $E_{\mathrm{LMG}}$ on the Bloch sphere. In these figures we mark different kinds of fixed points, namely local maxima $M_{l}$, global minima $m_{g}$ and saddle points $S$. References [4, 45] perform a detailed analysis of the classical energy of the LMG model with respect to the fixed points and present a phase diagram of the system. Reference 4$]$ finds an exact expression for the DOS in the thermodynamic limit, and shows that the fixed points of $E_{\mathrm{LMG}}$ are related to non-analyticities in the DOS. Therefore, a saddle point of the energy surface corresponds to a logarithmic singularity and a local maximum to a jump in the DOS.
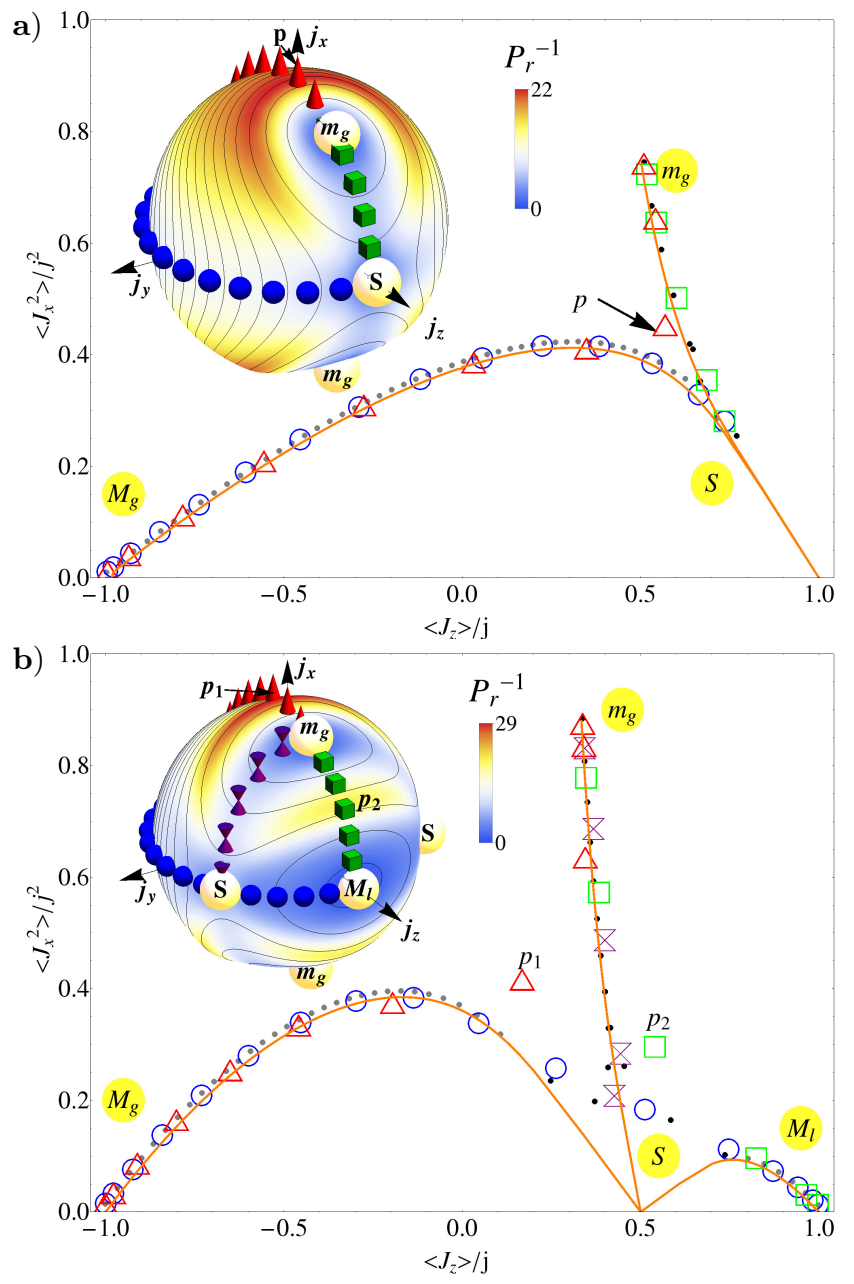

FIG. 1: (Color online) (a) Expectation values of $J_{z}$ and $J_{x}^{2}$ for $\gamma_{x} / h=2, \gamma_{y} / h=0$ and $j=30$ for the LMG model. (b) Same as in (a) but for $\gamma_{x} / h=3$ and $\gamma_{y} / h=2$. For each eigenstate $\left|E_{i}\right\rangle$ we calculate $\left\langle E_{i}\left|J_{z} / j\right| E_{i}\right\rangle$ and $\left\langle E_{i}\left|J_{x}^{2} / j^{2}\right| E_{i}\right\rangle$ and place a dot in the diagram. Black (gray) dots correspond to states with energy less (greater) than the saddle point energy. The solid orange line depicts the semiclassical calculation Eq. (7). The other symbols (triangles, squares, circles and double triangles) correspond to time-averaged expectation values Eq. 9 for $j=30$ and $\tau=20 / h$. The initial conditions are depicted on the Bloch sphere with the same shape as the symbols in the plot. We mark states centered at the global minimum, saddle point and local maximum with $m_{g}, S$ and $M_{l}$, respectively, and emphasize the points $p, p_{1}$ and $p_{2}$. Additionally, we show a density plot of the participation ratio $P_{r}^{-1}$ on the Bloch sphere. The black curves mark the classical trajectories with constant energy.

Due to the continuous symmetry $\hat{\mathcal{M}}$ of the TC model Eq. (2), we can derive an effective semiclassical energy landscape $E_{\mathrm{TC}}$ for the atomic ensemble.

Like for the LMG model, to obtain the semiclassical energy landscape, we scale the Hamiltonian Eq. (2) with $j$. This allows us to use the classical variables $\mathbf{j}=\left(j_{x}, j_{y}, j_{z}\right)$ for the atomic ensemble as in the LMG model. Furthermore, we use the classical variables $a, \vartheta \in \mathbb{R}$ for the 
bosonic mode defined by $a e^{i \vartheta}=\hat{a} / \sqrt{j}$. In addition, we define the classical conserved quantity $\mathcal{M}=\hat{\mathcal{M}} / j=$ $j_{z}+a^{2}$, which enables us to obtain the semiclassical energy landscape

$$
\begin{aligned}
E_{\mathrm{TC}}(\vartheta, \mathbf{j}) & =\omega \mathcal{M}+\left(\omega_{0}-\omega\right) j_{z} \\
& +\lambda \sqrt{2\left(\mathcal{M}-j_{z}\right)}\left(j_{x} \cos \vartheta-j_{y} \sin \vartheta\right) .
\end{aligned}
$$

Finally, we introduce rotated spin variables $\tilde{j}_{x}=$ $j_{x} \cos \vartheta-j_{y} \sin \vartheta, \tilde{j}_{y}=j_{x} \sin \vartheta+j_{y} \cos \vartheta$ and $\tilde{j}_{z}=j_{z}$. In terms of the new coordinates $\tilde{\mathbf{j}}=\left(\tilde{j}_{x}, \tilde{j}_{y}, \tilde{j}_{z}\right)$, the energy landscape

$$
E_{\mathrm{TC}}(\tilde{\mathbf{j}})=\omega \mathcal{M}+\left(\omega_{0}-\omega\right) \tilde{j}_{z}+\lambda \sqrt{2\left(\mathcal{M}-\tilde{j}_{z}\right)} \tilde{j}_{x}
$$

represents an effective energy landscape for the atomic ensemble. In the Appendix we show that the equations of motion of the rotated spins are given by $\frac{d}{d t} \tilde{j}_{\alpha}=$ $\left\{\tilde{j}_{\alpha}, E_{\mathrm{TC}}(\tilde{\mathbf{j}})\right\}$. The time evolution of the bosonic variables follows

$$
a^{2}=\mathcal{M}-\tilde{j}_{z} \quad \frac{d}{d t} \vartheta=-\omega-\frac{\lambda}{a \sqrt{2}} \tilde{j}_{x} .
$$

Consequently, the time evolution of $a, \vartheta$ depends on the rotated spins but not the other way around.

In the inset of Fig. 2 we depict the level sets of the energy landscape $E_{\mathrm{TC}}$ on the Bloch sphere. For $\mathcal{M}=$ 1 , the energy surface $E_{\mathrm{TC}}(\tilde{\mathbf{j}})$ possesses a saddle point, giving rise to an ESQPT [24, 43, 46].

\section{Time-averaged expectation values}

According to Refs. 4, 36, 47, the expectation value $\langle\hat{O}\rangle_{E}$ of an operator $\hat{O}$ in an eigenstate of the system can be related to the dynamics of the corresponding classical observable $o(t)$. At leading order in $1 / j$, such a relation reads

$$
\langle\hat{O}\rangle_{E}=\frac{1}{L} \sum_{l=1}^{L} \frac{1}{T_{l}(E)} \int_{0}^{T_{l}(E)} o^{(l)}(t) d t
$$

where $L$ denotes the number of connected trajectories defined by the relations $E=j E_{\mathrm{LMG}}(\mathbf{j})$ for the LMG model and $E=j E_{\mathrm{TC}}(\vartheta, \mathbf{j})$ for the TC model. In Eq. (7) we consider the representation $o^{(l)}(t)$ of the observable $o(t)$ restricted to the $l$-th trajectory with period $T_{l}(E)$. Furthermore, one has to sum over the trajectories $l$ in such a way that their union has the symmetry of the underlying system, i.e., the symmetries of Hamiltonians (1) and (2).

References 4, 5. show how to express the expectation values of observables in eigenstates in terms of the DOS. As a consequence, the expectation values inherit the singularities of the DOS.

Based on Eq. (7), we now suggest a method which could be used to experimentally detect a signature of the

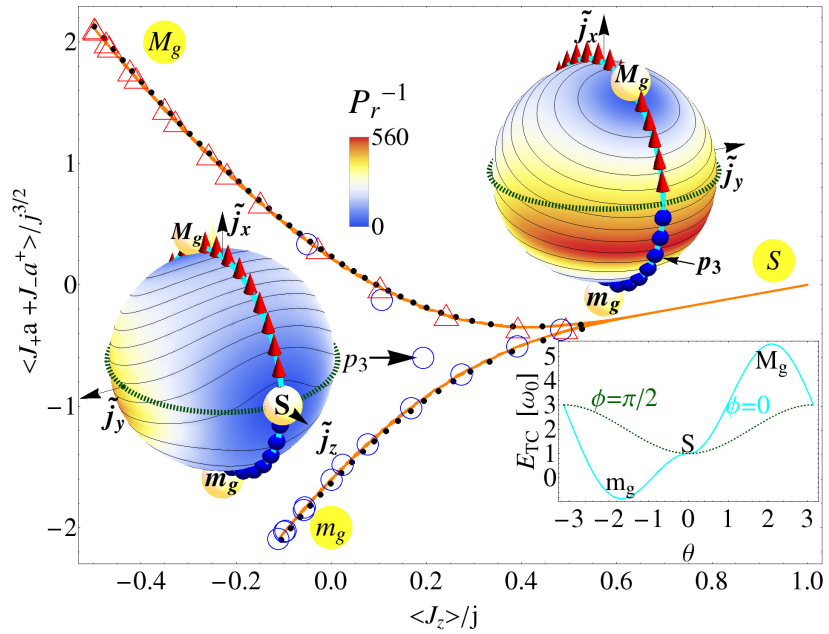

FIG. 2: (Color online) Expectation values in eigenstates (black dots) for the TC model (2) for the parameters $\omega / \omega_{0}=$ $\lambda / \omega_{0}=2, j=30$ and $M=30$. Time-averaged expectation values $(9)$ depicted as circles and triangles fulfill $\langle\hat{\mathcal{M}}\rangle=30$. The solid orange line depicts the semiclassical calculation (7). On the Bloch spheres the black lines depict the level sets of the energy landscape $E_{\mathrm{TC}}(\tilde{\mathbf{j}})$. The coloring shows the participation ratio $P_{r}^{-1}$ of the product of coherent states. The triangles and balls mark the positions of the initial states of our simulations. The light blue and green curve on the Bloch sphere mark the sections $\phi=0$ and $\phi=\pi / 2$, respectively. In the lower right inset we depict $E_{\mathrm{TC}}$ as a function of the local coordinates of the Bloch sphere $(\theta, \phi)$. In particular, we depict the dependence of the energy as a function of the polar angle $\theta$ for the azimuthal angles $\phi=0$ and $\phi=\pi / 2$. Here one can clearly see the saddle point at $\theta=0$.

ESQPT. The measurement of the energy of the system is often not experimentally accessible, which motivates us to employ an alternative representation of the observables. In order to resolve the singularities of measurable quantities, we choose two observables $\hat{O}_{1}$ and $\hat{O}_{2}$. In the numerical simulations we take $\left(\hat{O}_{1}, \hat{O}_{2}\right)=\left(\frac{J_{z}}{j}, \frac{J_{x}^{2}}{j^{2}}\right)$ for the LMG model and $\left(\hat{O}_{1}, \hat{O}_{2}\right)=\left(\frac{J_{z}}{j}, \frac{\hat{a} J_{+}+\hat{a}^{\dagger} J_{-}}{j^{3 / 2}}\right)$ for the TC model.

In contrast to the LMG model, the classical dynamics of the TC model is not restricted to a two-dimensional manifold. For this reason, we discuss the applicability of our method to this more complicated model below.

As spin-coherent states $|\theta, \phi\rangle$ are the closest ones to classical states [48, we take these as initial conditions for our quantum-mechanical simulations of the finite-size LMG model. A spin-coherent state is obtained by a rotation of the Dicke-State $|j, j\rangle$ so that its mean is located at the Bloch sphere coordinates $(\theta, \phi)$. More precisely, one can show that $|\theta, \phi\rangle=(1+|\rho|)^{-j} e^{\rho J_{-}}|j, j\rangle$, where $\rho=e^{i \phi} \tan \frac{\theta}{2}$. In the basis of Dicke states, a spin-coherent 
state reads $\sum_{m=-j}^{j} t_{m}|j, m\rangle$, where [49]

$$
t_{m}=\sqrt{\left(\begin{array}{c}
2 j \\
j+m
\end{array}\right)}\left(\sin \frac{\theta}{2}\right)^{j-m}\left(\cos \frac{\theta}{2}\right)^{j+m} e^{-i(j+m) \phi} .
$$

For the TC model, one can use a product of coherent states of both the spin system as well as the bosonic mode (see below for additional information).

We initially prepare the system in a (product of) coherent state $(\mathrm{s})|\psi(0)\rangle$. Afterwards, one measures the expectation values $\left\langle\hat{O}_{1}\right\rangle$ and $\left\langle\hat{O}_{2}\right\rangle$ in the state $|\psi(t)\rangle$, which allows us to define the temporal average

$$
\overline{\left\langle O_{i}\right\rangle}=\frac{1}{\tau} \int_{0}^{\tau}\left\langle\psi(t)\left|\hat{O}_{i}\right| \psi(t)\right\rangle d t
$$

where $\tau$ is the evolution time. This sequence is repeated for a set of different initial states. For a notational reason we define $\chi=\left(\left\langle O_{1}\right\rangle,\left\langle O_{2}\right\rangle\right)$. For the LMG model we specify $\chi_{(\theta, \phi)}$ at which the expectation values correspond to Eq. (9) with the initial state $|\theta, \phi\rangle$.

\section{RESULTS}

From an experimental point of view, an energyindependent representation of the expectation values has the big advantage that it is not necessary to measure the energy. Furthermore, such a representation would be useful to study systems in which energy is not a conserved quantity like in dissipative [50, 51] or in feed-back systems [52]. Thus, in doing so one can examine the implications of an ESQPT under nonequilibrium conditions.

\section{A. LMG model}

In Fig. 1 we compare the expectation values of observables in eigenstates, the semiclassical calculation found by using Eq. (7), and the quantum-mechanical averaging method Eq. (9). In the thermodynamic limit, the expectation values $\left\langle J_{z}\right\rangle$ and $\left\langle J_{x}^{2}\right\rangle$ calculated using Eq. (7) describe a parametric curve as a function of energy, which we denote with $\chi_{c l}(E)$. Accordingly, we denote the expectation values in eigenstates with $\chi_{e s}(E)$. In Fig. 1](a) there is a cusp of $\chi_{c l}(E)$ at $\chi=(1,0)$. This is a result of the ESQPT [4, as both expectation values exhibit a singular behavior there in the thermodynamic limit as a function of energy. The critical energy $E_{S}$ corresponds to the energy of the separatrix, which is $E_{S}=j E_{\mathrm{LMG}}(\mathbf{j})$ in Fig. 11 (a).

The expectation values of observables in eigenstates approximately agree with the semiclassical calculation. However, for finite sizes the expectation values in eigenstates are not directly located at $\chi=(1,0)$, because these points can be achieved only in the thermodynamic limit.
In Fig. 1 (b) the curve $\chi_{c l}(E)$ representing the semiclassical calculation exhibits a qualitatively different behavior. In contrast to Fig. 1(a) there is no cusp at the saddle point. Starting from the global minimum $m_{g}$ and increasing the energy $E$, the curve $\chi_{c l}(E)$ exhibits a bifurcation at the saddle point energy $E_{S}$. One branch continues to expectation values corresponding to the global maximum $M_{g}$ and the other one continues to expectation values corresponding to the local maximum $M_{l}$. Thus, this bifurcation is due to the emergence of a local maximum of the energy surface [4] which can be seen in the inset.

Most of the expectation values in eigenstates are well described by the classical calculation, but there are significant deviations for states close to the saddle point. States with energies less than the saddle point energy are nearly degenerate. Therefore, two states that are nearly degenerate have similar expectation values for the chosen observables. We color these expectation values black in Fig. 1, while the others are depicted in gray.

For the simulations of Eq. (9) we use the initial states depicted on the Bloch spheres in the insets of Fig. 1 (a) and (b). These are the most interesting initial states containing all fixed points of the energy landscape Eq. (3). Therefore, trajectories close to every possible energy in the system can be probed [26].

In Fig. 1(a), the finite-size simulation agrees with the semiclassical and eigenstate calculations. The curve $\chi_{(\theta, \phi)}$ exhibits a cusp close to $\chi=(1,0)$, although the energy of the spin-coherent state is smooth as a function of $(\theta, \phi)$. For this reason the path $\chi_{(\theta, \phi)}$ shows a signature of the non-analytic character of the ESQPT.

There is no point exactly located at the cusp at $\chi=$ $(1,0)$, although the system is initialized at the saddle point. This is a consequence of the quantum-mechanical deformation of the wave packet being prepared in the vicinity of the saddle point. The enhanced quantum fluctuations due to the influence of the saddle point cause a collapse and revival behavior in the time evolution of observables as it is discussed in Ref. [53. This also contributes to the deviation from the semiclassical calculation visible in Fig. 1 .

The deformation of the wave packet has been investigated experimentally in Refs. [30, 31, 54]. In contrast, wave packets remaining essentially Gaussian resemble the semiclassical calculation much more. Detailed investigations of the deviations of semiclassical and quantum dynamics can be found in Refs. [2, 9, 10, 22, [55. This argumentation also applies to Fig. 1(b), where the finitesize simulation is unable to resolve the bifurcation point $\chi=(1 / 2,0)$.

The initial states located at points $p, p_{1}$ and $p_{2}$ marked in the insets of Fig. 1 lying close to the separatrix also experience a strong deformation. Consequently, they considerably deviate from the semiclassical limit.

Deviations of the quantum-mechanical calculation from the semiclassical results are strongly related to the participation ratio $P_{r}^{-1}$ of the initial states $|\psi(0)\rangle=$ 
$|\theta, \phi\rangle$, with $|\theta, \phi\rangle$ being a spin-coherent state. Following Refs. 7, 56], we define the "inverse participation ratio" as $P_{r}=\sum_{i}\left|\left\langle\psi(0) \mid E_{i}\right\rangle\right|^{4}$, where $\left|E_{i}\right\rangle$ denotes an eigenstate of the system. The participation ratio is a measure of the number of eigenstates needed to construct a specific state. Therefore, a high participation ratio means that our initial state is a superposition of a lot of eigenstates.

In the insets of Fig. 1 we depict a density plot of $P_{r}^{-1}$ on the Bloch sphere. In particular, the initial points $p$, $p_{1}$ and $p_{2}$ in Fig. 1 which exhibit quite a strong deviation from the semiclassical calculation, have a high participation ratio. Consequently, the temporal average of these initial states is influenced by many eigenstates, so that it deviates strongly from the semiclassical calculation. The relation between the participation ratio and deviations of quantum dynamics is addressed in Refs. [2, 10, 22, 57. To improve the simulation close to $\chi=(1 / 2,0)$ in Fig. 1(b), we suggest new initial conditions, which we depict with purple double triangles in the inset. We choose therefore the points, at which the classical velocity is minimal [58, which possess a very low participation ratio.

The time-averaged expectation values $\overline{\left\langle O_{i}\right\rangle_{(\theta, \phi)}}$ converge to the corresponding semiclassical ones $\left\langle\hat{O}_{i}\right\rangle$ of Eq. 77). Given a finite size $j$, the scaling $\mid\left\langle\hat{O}_{i}\right\rangle-$ $\overline{\left\langle O_{i}\right\rangle_{(\theta, \phi)}} \mid \propto 1 / \log j$ for an initial state at a saddle point has been discussed in Ref. [2] for $\gamma_{y}=0$. We also checked this scaling numerically for $\gamma_{y} \neq 0$ and for other initial states at the separatrix .

\section{B. TC model}

For the TC model, Fig. 2 shows the expectation values of the observables $\hat{O}_{1}=J_{z} / j$ and $\hat{O}_{2}=\left(\hat{a} J_{+}+\right.$ $\left.\hat{a}^{\dagger} J_{-}\right) / j^{3 / 2}$ using the different calculation techniques. As explained before, the classical energy surface exhibits a saddle point for $M=j$. For this reason, black dots depict the observables in eigenstates $\chi_{e s}(E)$ for a finite-size system with $j=M=30$. Although the classical dynamics is not restricted to a two-dimensional manifold for the TC model, the expectation values in eigenstates can be calculated semiclassically with a high accuracy. The ESQPT cusp is located at $\boldsymbol{\chi}=(1,0)$. However, as for the LMG model, $\chi_{e s}(E)$ reaches this point only in the thermodynamic limit.

As stated before we suggest a product of spin and bosonic coherent states

$$
|\psi(0)\rangle=|\alpha\rangle \otimes|\theta, \phi\rangle
$$

as initial state, where $|\alpha\rangle$ denotes a coherent state of the bosonic mode [59]. Its mean photon number is given by $\left\langle\hat{a}^{\dagger} \hat{a}\right\rangle=|\alpha|^{2}$. In order to satisfy the condition $M=j$, the initial state $|\psi(0)\rangle$ shall fulfill

$$
\langle\hat{\mathcal{M}}\rangle=j \cos \theta+|\alpha|^{2}=j
$$

This constrain is also fulfilled by the expectation value of the time-evolved state as $\hat{\mathcal{M}}$ commutes with the Hamiltonian. Due to its definition, the initial state 110 is not restricted to the subspace $M=j$ and needs the whole Hilbert space to be defined. The variance of $\hat{\mathcal{M}}$ in the proposed initial state is $\operatorname{Var} \hat{\mathcal{M}}=j\left(1-\cos \theta+\frac{1}{2} \sin ^{2} \theta\right)$. Thus, in the thermodynamic limit the variance of the scaled quantity $\hat{\mathcal{M}} / j$ scales as $1 / j$.

We can use the symmetry $\hat{\mathcal{M}}$ to reduce the numerical effort. To this end, we decompose the initial state in a sum of states with different quantum numbers $M$. Therefore, we use the representation of spin and bosonic coherent states in terms of Fock and Dicke states, respectively [49. We write the initial state in Eq. 10p

$$
|\psi(0)\rangle=\sum_{M=M_{\min }}^{M_{\max }} \sum_{m=-j}^{\min (j, M)} a_{M-m} t_{m}|M-m\rangle \otimes|j, m\rangle,
$$

where $\left(M_{\min }, M_{\max }\right)=(-j, \infty)$,

$$
a_{n}=e^{-\frac{|\alpha|^{2}}{2}} \frac{\alpha^{n}}{\sqrt{n !}}
$$

and $t_{m}$ is given in 8 in terms of the symmetry-adapted basis discussed in Sec. III Therefore, $a_{n}$ and $t_{m}$ denote the coefficients of the bosonic and spin-coherent states, respectively. The amplitude of $\alpha$ is fixed due to the constrain (11). We choose the phase of $\alpha$ to be $\vartheta=\arg \alpha=0$, thus $\alpha=\sqrt{j-j \cos \theta}$. The time evolution for different $M$ for that initial state decouples due to the symmetry $\hat{\mathcal{M}}$, which reduces the numerical effort. In the numerical calculation we can truncate the state at $\left(M_{\min }, M_{\max }\right)=(j-\Delta M, j+\Delta M)$ with $\Delta M$ chosen in such a way that the time evolution of the expectation values converges.

Figure 2 depicts the results of the time-averaged quantum simulations. The initial conditions are sketched on the Bloch sphere with blue balls and red triangles located along the paths $\theta \in(0, \pi)$ for $\phi=0$ and $\phi=\pi$, respectively. The time-averaged expectation values are depicted with corresponding symbols. At this point we recall that the energy surface on the Bloch sphere is depicted in a rotated frame for which the rotation angle is given by $\vartheta$. As we choose $\vartheta=0$ for our initial conditions, the rotated frame is equivalent to the laboratory frame.

The result of the finite-size simulations resembles the findings for the LMG model in Fig. 1](a). The initial conditions close to the saddle point of the classical energy surface reproduce the expectation values of the semiclassical calculation with a high accuracy. However, for an initial condition located on the separatrix but away from the saddle point, there are also significant deviations from the semiclassical calculation. In Fig. 2 we denote this point with $p_{3}$. We also find that this initial condition is characterized by a high participation ratio $P_{r}^{-1}$, which smoothens the signature of the ESQPT. 


\section{APPLICATIONS}

Finally, we discuss the experimental applicability of our method for the LMG model. Here, we refer to the experimental realization of the LMG model in Refs. 30, 31. This experimental realization allows us to prepare the system in a spin-coherent state on arbitrary positions on the Bloch sphere [60. The measurement of the expectation values of $J_{z}$ and $J_{x}^{2}$ is performed by repeating the time evolution for the same initial state up to a given time $t$. Based on the single measurements one can then calculate the desired expectation value at time $t$.

The maximum feasible time $\tau$ should be long enough to observe recurrences in the time evolution of observables for all initial states [60]. In Fig. 3 we simulate these experimental circumstances for an experimentally feasible particle number $N=300$ and $\gamma_{x} / h=2$. Based on the realization of the LMG model in Ref. [30, 31, experimentally feasible parameters are $h=2 \pi \times 9.45 \mathrm{~Hz}$ and $\gamma_{x}=2 \pi \times 18.9 \mathrm{~Hz}$. From the time evolution of $\left\langle J_{z}(t)\right\rangle$ and $\left\langle J_{x}^{2}(t)\right\rangle$ we estimate the duration of one period $\tau$ for each initial preparation. As examples we depict in Fig. 3(a) the time evolution of an initial state located at the saddle point and of an initial state located close to the global minimum. We also mark the respective estimated period $\tau$. In the inset of Fig. 3(b) we show the chosen $\tau$ for the initial states depicted in the inset of Fig. 11(a). As continuous measurements are not possible, we consider a discretized version of Eq. (9), namely

$$
{\overline{\left\langle O_{i}\right\rangle}}_{(\theta, \phi)}=\frac{1}{\tau} \sum_{k=0}^{n_{(\theta, \phi)}-1}\left\langle\psi\left(t_{k}\right)\left|\hat{O}_{i}\right| \psi\left(t_{k}\right)\right\rangle \Delta t
$$

where the time step $\Delta t$ and $n_{(\theta, \phi)}$ fulfill $\Delta t n_{(\theta, \phi)}=\tau$ and $t_{k}=k \Delta t$. To minimize the experimental effort it will be convenient to take $\Delta t$ as large as possible. In Fig. 3 we choose $\Delta t=\frac{1}{4 h}$, which still enables a sufficient precision. For the parameters given above this means that $\tau \leq 134 \mathrm{~ms}$ and $\Delta t=4.2 \mathrm{~ms}$, so that at most $n_{(\theta, \phi)}=32$ for an initial state at the saddle point. As a demonstration, in Fig. 3(a) we mark the points used in the average (14) with dots. Due to our choice of $\Delta t$ these points are dense in relation to the temporal variation of the observables. As the chosen $\tau$ are quite small, the average does not suffer from the complex collapse and revival behavior appearing for longer evolution times observed in Ref. 53.

As explained before, depending on the initial condition the state can be strongly deformed. This hinders the measurement of the expectation values as one has to repeat the measurement more often to obtain the required precision. To estimate this effort, we also included bars for each point in Fig. 3(b) depicting the corresponding time-averaged variance of the time evolution,

$$
\overline{\operatorname{Var} \hat{O}_{i}}=\frac{1}{\tau} \sum_{k=0}^{n_{(\theta, \phi)}-1} \operatorname{Var} \hat{O}_{i}\left(t_{k}\right) \Delta t
$$
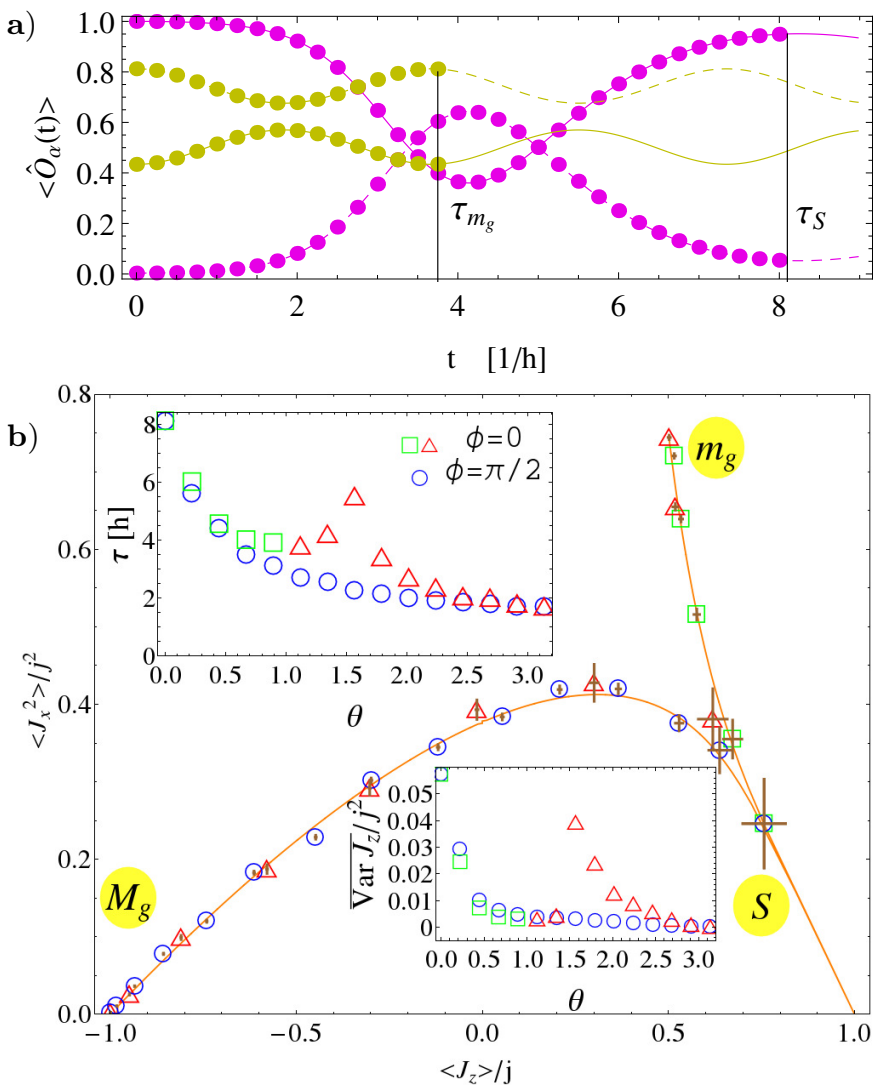

FIG. 3: (Color online) (a) Example of the time evolution of $\hat{O}_{1}=J_{z} / j$ (solid lines) and $\hat{O}_{2}=J_{x}^{2} / j^{2}$ (dashed lines) in the LMG model for initial states located at the saddle point [pink (dark gray)] and close to a global minimum [yellow (light gray)]. Parameters are the same as in Fig. 1(a), but $j=150$. The black lines depict the estimated periods $\tau_{S, m_{g}}$ for the chosen initial states, respectively. The dots mark the times $t_{k}$ used to evaluate Eq. (14) at which the time step is chosen to be $\Delta t=\frac{1}{4 h}$. (b) Realistic simulation of the method for the LMG model. The colored symbols and the orange line depict the same as in Fig. 1(a). The period $\tau$ is always estimated by the time evolution of the observables and depicted in the top inset. Here we also used $\Delta t=\frac{1}{4 h}$. The bars depict the timeaveraged variance of the observables which is defined in 15 . In the bottom inset we depict the time-averaged variance of $J_{z}$.

where

$$
\operatorname{Var} \hat{O}_{i}(t)=\left\langle\psi(t)\left|\left(\hat{O}_{i}-\left\langle\hat{O}_{i}(t)\right\rangle\right)^{2}\right| \psi(t)\right\rangle
$$

is the variance of the observable $\hat{O}_{i}$ at time $t$. We depict the time-averaged variance of $\hat{O}_{i}=J_{z}$ in the inset of Fig. 3(b). We see that for initial states away from the saddle point the variance is vanishing small, as the states remain essentially Gaussian, but for states close to the separatrix one has to repeat the time evolution for a given time $t$ quite often.

The time-averaged variance defined in Eq. 15 does account only for the quantum fluctuations of the system. However, the actual uncertainties appearing in our 
method depend on the experimental realization. For example, one could also consider other influences, e.g., uncertainties in preparing the initial state. However, in the context of the experimental realization of Ref. [30, 31] we assume this to have a minor influence on the measured results, as there is a high degree of control of the preparation of the initial state.

Referring to Ref. 31], we assume that about $n_{\mathrm{EM}}=60$ experimental measurements are sufficient to determine adequately the expectation values of $\hat{O}_{i}$ for each time step in Fig. 3(a). Consequently, to obtain the point in Fig. 3(b) corresponding to the saddle point, one has to perform $n_{\mathrm{SEM}}=2 n_{\mathrm{EM}} n_{(\theta, \phi)}=3840$ single experimental measurements, where $n_{(\theta, \phi)}=32$ for the saddle point as in Eq. (14). We note that in the experimental realization presented in Ref. 61] up to $n_{\mathrm{PM}}=30$ measurements can be performed in parallel in one experimental run so that one needs $n_{\mathrm{SEM}} / n_{\mathrm{PM}}=128$ experimental runs. For the other points in Fig. 3(b) fewer experimental runs are necessary.

\section{CONCLUSIONS}

Expectation values of quantum-mechanical operators in eigenstates can be calculated using the classical dynamics of the system. This motivated us to suggest a method which opens a new avenue to experimentally detect signatures of the ESQPT in systems in which energy is not experimentally accessible or not conserved.

The temporal averaging of a finite-size system resembles the expectation values in eigenstates and the semiclassical calculations. However, there are partial deviations from the semiclassical calculations for energies near an ESQPT. These deviations are bigger for initial states obeying a high participation ratio.

A point to be addressed in the future is the application of our method to more complicated mean-field type models such as spinor Bose-Einstein condensates [54, 62 . and the Dicke model [33, 34. In particular, our representation of observables might be interesting in the description of nonequilibrium systems, in which the energy is not a conserved quantity, such as driven [63, 64, dissipative 50, 51] or feedback [52] mean field-type systems.

\section{Acknowledgments}

The authors gratefully acknowledge financial support from the DFG Grants BR 1528/7, BR 1528/8, BR 1528/9, SFB 910 and GRK 1558 and inspiring conversations with $\mathrm{P}$. Pérez-Fernández, M. Vogl and M. Oberthaler and the members of his group.
[1] C. Emary and T. Brandes, Physical Review E 67, 066203 (2003).

[2] M. Chuchem, K. Smith-Mannschott, M. Hiller, T. Kottos, A. Vardi, and D. Cohen, Phys. Rev. A 82, 053617 (2010).

[3] M. Caprio, P. Cejnar, and F. Iachello, Ann. Phys. (N.Y.) 323, 1106 (2008).

[4] P. Ribeiro, J. Vidal, and R. Mosseri, Phys. Rev. E 78, 021106 (2008).

[5] T. Brandes, Phys. Rev. E 88, 032133 (2013).

[6] R. Puebla, A. Relaño, and J. Retamosa, Phys. Rev. A 87, 023819 (2013).

[7] R. Scharf and B. Sundaram, Phys. Rev. A 45, 3615 (1992).

[8] S. Aubry, S. Flach, K. Kladko, and E. Olbrich, Phys. Rev. Lett. 76, 1607 (1996).

[9] F. Nissen and J. Keeling, Phys. Rev. A 81, 063628 (2010).

[10] S. F. Caballero-Bentez, V. Romero-Rochn, and R. Paredes, J. Phys. B 43, 115301 (2010).

[11] B. Juliá-Díaz, J. Martorell, and A. Polls, Phys. Rev. A 81, 063625 (2010).

[12] B. Juliá-Díaz, D. Dagnino, M. Lewenstein, J. Martorell, and A. Polls, Phys. Rev. A 81, 023615 (2010).

[13] Y. Kawaguchi and M. Ueda, Phys. Rep. 520, 253 (2012).

[14] L. Bernstein, Phys. D (Amsterdam, Neth.) 68, 174 (1993).

[15] R. Franzosi, V. Penna, and R. Zecchina, Int. J. Mod. Phys. B 14, 943 (2000).

[16] R. Franzosi and V. Penna, Phys. Rev. A 63, 043609 (2001).
[17] Z. Karkuszewski, K. Sacha, and A. Smerzi, Eur. Phys. J. D 21, 251 (2002).

[18] P. Buonsante, R. Franzosi, and V. Penna, J. Phys. B 37, S229 (2004).

[19] E. M. Graefe and H. J. Korsch, Phys. Rev. A 76, 032116 (2007).

[20] B. Juliá-Díaz, E. Torrontegui, J. Martorell, J. G. Muga, and A. Polls, Phys. Rev. A 86, 063623 (2012).

[21] B. Juliá-Díaz, T. Zibold, M. K. Oberthaler, M. MeléMesseguer, J. Martorell, and A. Polls, Phys. Rev. A 86, 023615 (2012).

[22] H. Hennig, D. Witthaut, and D. K. Campbell, Phys. Rev. A 86, 051604 (2012).

[23] P. Pérez-Fernández, A. Relaño, J. M. Arias, P. Cejnar, J. Dukelsky, and J. E. García-Ramos, Phys. Rev. E 83, 046208 (2011).

[24] P. Pérez-Fernández, P. Cejnar, J. M. Arias, J. Dukelsky, J. E. García-Ramos, and A. Relaño, Phys. Rev. A 83, 033802 (2011).

[25] P. Pérez-Fernández, A. Relaño, J. M. Arias, J. Dukelsky, and J. E. García-Ramos, Phys. Rev. A 80, 032111 (2009).

[26] V. M. Bastidas, P. Pérez-Fernández, M. Vogl, and T. Brandes, Phys. Rev. Lett. 112, 140408 (2014).

[27] B. P. Winnewisser, M. Winnewisser, I. R. Medvedev, M. Behnke, F. C. De Lucia, S. C. Ross, and J. Koput, Phys. Rev. Lett. 95, 243002 (2005).

[28] L. Zhao, J. Jiang, T. Tang, M. Webb, and Y. Liu, Phys. Rev. A 89, 023608 (2014).

[29] B. Dietz, F. Iachello, M. Miski-Oglu, N. Pietralla, A. Richter, L. von Smekal, and J. Wambach, Phys. Rev. 
B 88, 104101 (2013).

[30] T. Zibold, E. Nicklas, C. Gross, and M. K. Oberthaler, Phys. Rev. Lett. 105, 204101 (2010).

[31] C. Gross, T. Zibold, E. Nicklas, J. Estève, and M. K. Oberthaler, Nature (London) 464, 1165 (2010).

[32] M. J. Steel and M. J. Collett, Phys. Rev. A 57, 2920 (1998).

[33] K. Baumann, C. Guerling, F. Brennecke, and T. Esslinger, Nature (London) 464, 1301 (2010).

[34] K. Baumann, R. Mottl, F. Brennecke, and T. Esslinger, Phys. Rev. Lett. 107, 140402 (2011).

[35] M. P. Baden, K. J. Arnold, A. L. Grimsmo, S. Parkins, and M. D. Barrett, Phys. Rev. Lett. 113, 020408 (2014).

[36] T. Paul and A. Uribe, Ann. I.H.P. Phys. Theor. 59, 357 (1993).

[37] H. Lipkin, N. Meshkov, and A. Glick, Nuclear Physics 62, 188 (1965).

[38] N. Meshkov, A. Glick, and H. Lipkin, Nuclear Physics 62, 199 (1965).

[39] A. Glick, H. Lipkin, and N. Meshkov, Nuclear Physics 62, 211 (1965).

[40] R. H. Dicke, Phys. Rev. 93, 99 (1954).

[41] J. Keeling, Phys. Rev. A 79, 053825 (2009).

[42] M. Tavis and F. W. Cummings, Phys. Rev. 170, 379 (1968).

[43] L. M. Narducci, M. Orszag, and R. A. Tuft, Phys. Rev. A 8, 1892 (1973).

[44] S. Dusuel and J. Vidal, Phys. Rev. B 71, 224420 (2005).

[45] O. Castaños, R. López-Peña, J. G. Hirsch, and E. LópezMoreno, Phys. Rev. B 74, 104118 (2006).

[46] M. A. Bastarrachea-Magnani, S. Lerma-Hernández, and J. G. Hirsch, Phys. Rev. A 89, 032102 (2014).

[47] P. Ribeiro and T. Paul, Phys. Rev. A 79, 032107 (2009).

[48] F. T. Arecchi, E. Courtens, R. Gilmore, and H. Thomas, Phys. Rev. A 6, 2211 (1972).

[49] W.-M. Zhang, D. H. Feng, and R. Gilmore, Rev. Mod. Phys. 62, 867 (1990).

[50] S. Morrison and A. S. Parkins, Phys. Rev. Lett. 100, 040403 (2008).

[51] W. Kopylov, C. Emary, and T. Brandes, Phys. Rev. A 87, 043840 (2013).

[52] W. Kopylov, C. Emary, E. Schöll, and T. Brandes, New J. Phys. 17, 013040 (2015).

[53] A. P. Tonel, J. Links, and A. Foerster, J. Phys. A 38, 1235 (2005).

[54] C. Gerving, T. Hoang, B. Land, M. Anquez, C. Hamley, and M. Chapman, Nat. Commun. 3, 1169 (2012).

[55] A. Buchleitner, D. Delande, and J. Zakrzewski, Phys. Rep. 368, 409 (2002).

[56] D. Weaire and V. Srivastava, J. Phys. C 10, 4309 (1977).

[57] C. Khripkov, D. Cohen, and A. Vardi, J. Phys. A 46, 165304 (2013).

[58] M. V. Berry and K. E. Mount, Rep. Prog. Phys. 35, 315 (1972).

[59] R. J. Glauber, Phys. Rev. 131, 2766 (1963).

[60] Oberthaler et al., (private communications).

[61] W. Muessel, H. Strobel, D. Linnemann, D. B. Hume, and M. K. Oberthaler, Phys. Rev. Lett. 113, 103004 (2014).

[62] C. Hamley, C. Gerving, T. Hoang, E. Bookjans, and M. Chapman, Nat. Phys. 8, 305 (2012).

[63] V. M. Bastidas, C. Emary, B. Regler, and T. Brandes, Phys. Rev. Lett. 108, 043003 (2012).

[64] G. Engelhardt, V. M. Bastidas, C. Emary, and T. Brandes, Phys. Rev. E 87, 052110 (2013).

\section{Appendix A: Classical equation of motion for the Tavis-Cummings model}

A semiclassical investigation of the TC model has been performed in Refs. 24, 46. Here we present a different approach to illustrate the semiclassical dynamics.

The Heisenberg equations of motion of the operators in the TC model read

$$
\begin{aligned}
\frac{d}{d t} \hat{a} & =-i \omega \hat{a}-i \frac{\lambda}{\sqrt{N}} J_{-} \\
\frac{d}{d t} J_{x} & =-\omega_{0} J_{y}-\frac{\lambda}{\sqrt{N}} \frac{1}{i}\left(\hat{a}-\hat{a}^{\dagger}\right) J_{z} \\
\frac{d}{d t} J_{y} & =\omega_{0} J_{x}-\frac{\lambda}{\sqrt{N}}\left(\hat{a}+\hat{a}^{\dagger}\right) J_{z} \\
\frac{d}{d t} J_{z} & =-i \frac{\lambda}{\sqrt{N}}\left(\hat{a} J_{+}-\hat{a}^{\dagger} J_{-}\right) .
\end{aligned}
$$

In the thermodynamic limit $j \rightarrow \infty$, we consider the equations of motion for the classical variables $a e^{i \vartheta}=$ $\hat{a} / \sqrt{j}$ and $j_{\alpha}=J_{\alpha} / j$, with $a, \vartheta, j_{\alpha} \in \mathbb{R}$. The equations of motion of these new coordinates then read

$$
\begin{aligned}
\frac{d}{d t} a & =-\frac{\lambda}{\sqrt{2}}\left(\sin \vartheta j_{x}+\cos \vartheta j_{y}\right) \\
\frac{d}{d t} \vartheta & =-\omega-\frac{\lambda}{\sqrt{2} a}\left(\cos \vartheta j_{x}-\sin \vartheta j_{y}\right) \\
\frac{d}{d t} j_{x} & =-\omega_{0} j_{y}-\lambda \sqrt{2} a \sin \vartheta j_{z} \\
\frac{d}{d t} j_{y} & =\omega_{0} j_{x}-\lambda \sqrt{2} a \cos \vartheta j_{z} \\
\frac{d}{d t} j_{z} & =\lambda \sqrt{2} a\left(\sin \vartheta j_{x}+\cos \vartheta j_{y}\right)
\end{aligned}
$$

In these new coordinates, the particle conservation in the thermodynamic limit is

$$
\mathcal{M} \equiv \lim _{j \rightarrow \infty} \frac{\hat{M}}{j}=j_{z}+a^{2} .
$$

Due to this conserved quantity, there is a constrain for the solutions $a$ and $j_{z}$. We define the rotated angular momentum $\tilde{j}_{x}=j_{x} \cos \vartheta-j_{y} \sin \vartheta, \tilde{j}_{y}=j_{x} \sin \vartheta+j_{y} \cos \vartheta$ and $\tilde{j}_{z}=j_{z}$. After some algebraic manipulations of Eqs. A5 - A9 , we obtain the equations of motion for the rotated angular momentum. By using the equations of motion for the classical variables $a, \vartheta$ describing the bosonic mode, Eqs. A10 and (A6), we obtain

$$
\begin{aligned}
\frac{d}{d t} \tilde{j}_{x} & =-\omega_{0} \tilde{j}_{y}-\left(-\omega-\frac{\lambda}{\sqrt{2\left(\mathcal{M}-\tilde{j}_{z}\right)}} \tilde{j}_{x}\right) \tilde{j}_{y} \\
\frac{d}{d t} \tilde{j}_{y} & =\omega_{0} \tilde{j}_{x}-\lambda \sqrt{2\left(\mathcal{M}-\tilde{j}_{z}\right)} \tilde{j}_{z} \\
& +\left(-\omega-\frac{\lambda}{\sqrt{2\left(\mathcal{M}-\tilde{j}_{z}\right)}} \tilde{j}_{x}\right) \tilde{j}_{x} \\
\frac{d}{d t} \tilde{j}_{z} & =\lambda \sqrt{2\left(\mathcal{M}-\tilde{j}_{z}\right)} \tilde{j}_{y}
\end{aligned}
$$


These equations of motion can be directly derived starting from the effective energy landscape

$$
E_{T C}(\tilde{\mathbf{j}})=\omega\left(\mathcal{M}-\tilde{j}_{z}\right)+\omega_{0} \tilde{j}_{z}+\lambda \sqrt{2\left(M-\tilde{j}_{z}\right)} \tilde{j}_{x}
$$

by evaluating the Poisson brackets, namely, $\frac{d}{d t} \vec{j}_{\alpha}=$ $\left\{\tilde{j}_{\alpha}, E_{\mathrm{TC}}(\tilde{\mathbf{j}})\right\}$. For this derivation one has to take into account the following Poisson bracket relations:

$$
\begin{aligned}
& \left\{\sqrt{2\left(\mathcal{M}-\tilde{j}_{z}\right)}, \tilde{j}_{x}\right\}=\frac{-\tilde{j}_{y}}{\sqrt{2\left(\mathcal{M}-\tilde{j}_{z}\right)}} \\
& \left\{\sqrt{2\left(\mathcal{M}-\tilde{j}_{z}\right)}, \tilde{j}_{y}\right\}=\frac{\tilde{j}_{x}}{\sqrt{2\left(\mathcal{M}-\tilde{j}_{z}\right)}} .
\end{aligned}
$$

These relations can be derived by representing the angular momentum operators with the Cartesian position and momentum operators. 